\title{
UNIFORMIDADE DE DISTRIBUIÇÃO VOLUMÉTRICA DE PONTAS DE PULVERIZAÇÃO HIDRÁULICA EM DIFERENTES CONDIÇÕES OPERACIONAIS
}

Robson Shigueaki Sasaki ${ }^{1}$, Mauri Martins Teixeira ${ }^{2}$, Cleyton Batista de Alvarenga ${ }^{3}$, André Luis da Silva Quirino ${ }^{4}$, Rafael Augusto Soares Tiburcio ${ }^{5}$

\begin{abstract}
RESUMO
$\mathrm{Na}$ aplicação de agrotóxicos, os equipamentos que se baseiam na pulverização hidráulica são os mais difundidos, graças à flexibilidade que oferecem em diferentes situações. A ponta de pulverização é um componente fundamental para proporcionar uma boa uniformidade de distribuição e espectro de gotas adequado. A uniformidade de distribuição é caracterizada pelo coeficiente de variação $(\mathrm{CV})$ da distribuição volumétrica superficial, sendo ambos inversamente proporcionais. Objetivou-se, com este trabalho, avaliar quatro diferentes pontas de pulverização (XR 110.02 VK, AXI ISO 110.03, AXI ISO 110.04 e AXI ISO 110.05), sob três alturas da barra em relação ao alvo ( 0,$4 ; 0,5$ e $0,6 \mathrm{~m})$ e em três pressões de trabalho $(200,300$, e $400 \mathrm{kPa})$. Observou-se que a altura e a pressão de trabalho influenciaram na uniformidade de distribuição do líquido. A melhor condição de trabalho para a ponta XR $110.02 \mathrm{VK}$, foi a $0,4 \mathrm{~m}$ e $400 \mathrm{kPa}$, com CV de 8,5\%. Para os modelos AXI ISO 110.03 e AXI ISO 110.04, foram a 0,4 m e $300 \mathrm{kPa}$, com CV de 7,1 \% e 6,3\%, respectivamente, e para o modelo AXI ISO 110.05 JACTO, foi a 0,5 m e pressão de $200 \mathrm{kPa}$, com CV de $7,7 \%$.
\end{abstract}

Palavras-chave: bico de pulverização, coeficiente de variação, aplicação de agrotóxicos

\section{ABSTRACT \\ VOLUME UNIFORMITY DISTRIBUTION OF HYDRAULIC SPRAY NOZZLES UNDER DIFFERENT WORKING CONDITIONS}

Hydraulic spray equipments are commonly used for pesticide application due to their flexibility under different situations. The spray nozzle is the key component to ensure distribution uniformity and suitable spectrum of droplets. The distribution uniformity is characterized by the coefficient of variation (CV) of the surface volumetric distribution, which are inversely proportional. This study was done to evaluate four different nozzles (XR $11002 \mathrm{VK}$, AXI ISO 110.03, AXI ISO 110.04 e AXI ISO 110.05), with spray boom height at $0.4,0.5$ or $0.6 \mathrm{~m}$ from the target, at the operating pressure of 200,300 , or $400 \mathrm{kPa}$. The data showed that spray boom height and pressure influenced the distribution uniformity of the liquid. The most appropriate height-pressure combination for the XR11002VK was 0.4 m-400 kPa, with CV of $8.5 \%$, for AXIISO110.03 and AXIISO110.04 was $0.4 \mathrm{~m}-300 \mathrm{kPa}$, with CV of $7.1 \%$ and $6.3 \%$, respectively, while for AXI ISO 11005 JACTO the best was $0.5 \mathrm{~m}-200 \mathrm{kPa}$ with CV of 7.7\%.

Keywords: nozzle AXI model, coefficient of variation, pesticide spray

\section{Recebido para publicação em 25/02/2011. Aprovado em 01/09/2011.}

1- Eng. Agrônomo, Mestrando em Engenharia Agrícola, Bolsista Capes, Dep. Eng. Agrícola, UFV - Viçosa - MG, email: robsonsasaki@ yahoo.com.br;

2- Professor Adjunto, Departamento de Engenharia Agrícola, UFV, email: mauri@ufv.br;

3 - Eng. Agrônomo, Doutorando em Engenharia Agrícola, Bolsista CNPq, UFV - Viçosa - MG, email: cleytonbatista@yahoo.com.br;

4- Eng. Agrônomo, Doutorando em Engenharia Agrícola, Bolsista Capes, UFV - Viçosa - MG, email: andrelsquirino@yahoo.com.br;

5- Eng. Florestal, Doutorando em Engenharia Agrícola, UFV - Viçosa-MG, email: rafael_tiburcio@hotmail.com. 


\section{INTRODUÇÃO}

O objetivo da aplicação de agrotóxicos é o controle de insetos, patógenos e plantas daninhas prejudiciais às culturas. A aplicação de agrotóxicos deve ser uma operação racional e técnica, reduzindo a contaminação ambiental, os danos à saúde do trabalhador e a seleção de biótipos resistentes (BALAN et al., 2008).

Entre as diferentes técnicas de aplicação de agrotóxicos, as que se baseiam na pulverização hidráulica são as mais difundidas, graças à sua flexibilidade em diferentes situações. Nesses equipamentos, as pontas de pulverização são componentes fundamentais, pois influenciam diretamente na qualidade e na segurança da aplicação. A seleção das pontas é um dos fatores mais importantes para uma aplicação eficiente e de qualidade, permitindo o controle preciso da vazão, porcentagem de cobertura e da distribuição da calda sobre o alvo (FERNANDES et al., 2007).

Nas aplicações de agrotóxicos, é importante estabelecer alguns parâmetros para avaliar e controlar a técnica utilizada, garantindo a qualidade do tratamento com mínimo efeito danoso ao homem e ao ambiente (RODRIGUES et al., 2008). Há necessidade de redução das doses e do volume de aplicação para a redução dos custos de produção, devendo ser alcançada a uniformidade na distribuição da calda em toda a barra durante a pulverização, caso contrário a dose depositada no alvo poderá ser insuficiente para gerar o efeito biológico desejável no controle de pragas e doenças (BAUER et al., 2006). Segundo Cunha e Ruas (2006), uma cobertura homogênea na pulverização pressupõe uma distribuição uniforme de líquidos, caracterizada por baixos coeficientes de variação da distribuição volumétrica superficial, tanto no sentido longitudinal, como no transversal.

Uma das formas de quantificar a uniformidade de distribuição da pulverização é por meio da análise de deposição do produto na área, expressa pelo coeficiente de variação $(\mathrm{CV})$. Quanto maior o coeficiente de variação, maior a variação da distribuição e menor será a uniformidade da aplicação. Os padrões adequados de CV são de 10 a $15 \%$ (WOLF, SMITH, 1979; DeBOER, WIENS, 1983; FAO, 1997), sendo que valores acima desse limite podem ser indicativos de pontas de pulverização desgastadas, pontas diferentes na barra, espaçamento variado entre bicos ou má qualidade das pontas. Na Europa, em ensaio de laboratório, para as pressões e alturas recomendadas pelos fabricantes, o coeficiente de variação deve ser inferior a $7 \%$, e para as demais pressões e alturas especificadas pelo fabricante como passíveis de uso, o coeficiente de variação não deve exceder 9 $\%$ (FAQIRI; KRISHNAN, 2001).

Em pulverizadores hidráulicos de barra, a uniformidade na distribuição transversal é condicionada pela altura da barra, pressão de trabalho, vazão das pontas de pulverização, entre outros fatores (BARCELLOS et al., 2006).

Deste modo, objetivou-se determinar a uniformidade de distribuição das pontas XR 110.02 VK, AXI ISO 110.03, AXI ISO 110.04 e AXI ISO 110.05 em função da altura da barra e da pressão de trabalho.

\section{MATERIAL E MÉTODOS}

O trabalho foi conduzido no Laboratório de Mecanização do Departamento de Engenharia Agrícola da Universidade Federal de Viçosa, Minas Gerais, cujas condições ambientais permaneceram com temperatura entre 25 e $28^{\circ} \mathrm{C}$, umidade relativa entre 70 e $75 \%$ e ausência de vento.

$\mathrm{O}$ ensaio foi realizado em esquema fatorial $4 \times 3 \times 3$, constituídopor quatropontas de pulverização (XR 110.02 VK TEEJET, AXI ISO 110.03 JACTO, AXI ISO 110.04 JACTO e AXI ISO 110.05 JACTO), três alturas da barra de pulverização $(0,4$; $0,5$ e $0,6 \mathrm{~m})$ e três pressões de trabalhos $(200,300$ e $400 \mathrm{kPa}$ ), totalizando 36 tratamentos, estudados em delineamento inteiramente casualizado, com quatro repetições.

As pontas XR 110.02 VK TEEJET, AXI ISO 110.03 JACTO, AXI ISO 110.04 JACTO e AXI ISO 110.05 JACTO são de perfil de jato plano e foram estudadas com espaçamento entre bicos de $0,50 \mathrm{~m}$ e pressões de trabalho dentro das recomendações dos fabricantes.

Para a determinação da uniformidade de distribuição de líquidos, as pontas foram montadas em uma bancada padronizada, conforme a norma ISO 5682/1 (ISO, 1986). A mesa padrão apresenta

\section{REVENG}

ENGENHARIA NA AGRICULTURA, VIÇOSA - MG, V.19 N.6, NOVEMBRO / DEZEMBRO 2011 
as dimensões de dois metros de comprimento por um metro de largura, canaletas em forma de "V" com $0,05 \mathrm{~m}$ de profundidade e largura, acionada por uma bomba de pistão com motor elétrico de $220 \mathrm{~V}$ e $2,2 \mathrm{~kW}$ de potência, apresentando um manômetro (com precisão de $13,79 \mathrm{kPa}$ ) e 32 provetas com precisão de $2 \mathrm{~mL}$.

A exatidão do manômetro foi aferida com auxílio de uma bomba de calibração, dotada de um manômetro de precisão (Classe A3), instalada em uma tubulação comunicante com o manômetro da bancada, sendo a tubulação ligada a um cilindro contendo um êmbolo. Este êmbolo é acionado manualmente com auxílio de uma manivela, que gira um parafuso. $\mathrm{O}$ movimento do parafuso bem como a posição do êmbolo determina o nível de pressurização de água ou silicone dentro do cilindro, comunicando pressão aos manômetros, possibilitando comparações entre ambos, conforme descrito por Alvarenga (2009).

Após a aferição do manômetro e anteriormente ao início do experimento, aferiu-se também a vazão nominal das pontas de pulverização, seguindo as normas de padronização ISO 10625. O teste para a verificação da vazão das pontas foi realizado com auxílio de uma proveta graduada com precisão de 20 $\mathrm{mL}$, coletando-se o líquido por um minuto na pressão nominal de $300 \mathrm{kPa}$. Utilizou-se um conjunto de três pontas de mesma vazão nominal para a determinação da uniformidade de distribuição.

As pontas foram instaladas na barra, e com auxílio de chave própria, fez-se o ajuste do ângulo do jato para evitar o choque entre jatos adjacentes, permitindo a sobreposição, conforme descrito por Ferreira et al. (2007).

A uniformidade de distribuição foi mensurada de acordo com o líquido coletado nas provetas da bancada de ensaio por meio do coeficiente de variação que foi calculado conforme a Equação 1 .

$$
\mathrm{CV}=\frac{\mathrm{s}}{\overline{\mathrm{x}}}
$$

em que

$\mathrm{CV}=$ coeficiente de variação $(\%)$;

$\mathrm{s}=$ desvio padrão $(\mathrm{mL}) ; \mathrm{e}$

$\overline{\mathbf{x}}=$ volume médio dos valores observados $(\mathrm{mL})$.

\section{RESULTADOS E DISCUSSÃO}

A aferição do manômetro possibilitou a obtenção de ajuste na pressão do líquido, ajustadas as correções na pressão do manômetro da bancada (Equação 2). O coeficiente de determinação foi de 0,9998, demonstrando que $99,98 \%$ das variações em Y são explicadas pelas variações em X.

$$
y=0,994 x-17,20
$$

em que

$\mathrm{x}=$ Pressão no manômetro padrão, $(\mathrm{kPa})$; e $\mathrm{y}=$ Pressão no manômetro na mesa de ensaios, $(\mathrm{kPa})$.

$\mathrm{Na}$ avaliação da vazão nominal, verificouse que todas as pontas estavam de acordo com a norma ISO 10625. Avaliou-se o coeficiente de variação entre as médias das vazões proporcionadas pelas pontas, sendo que o $\mathrm{CV}$ máximo obtido foi de $2,49 \%$, demonstrado que todas as pontas apresentam uma boa repetitividade quanto à vazão. A média da vazão nominal das pontas $\left(\mathrm{P}_{1}, \mathrm{P}_{2}\right.$ e $\left.\mathrm{P}_{3}\right)$ foi testada na pressão nominal de trabalho (Quadro 1).

Quadro 1.Vazão média das pontas de pulverização na pressão de $300 \mathrm{kPa}$

\begin{tabular}{ccccc}
\hline \multirow{2}{*}{ Ponta } & $\begin{array}{c}\text { Vazão } \\
\text { Nominal }\end{array}$ & $\mathrm{P}_{1}$ & $\begin{array}{c}\text { Pontas } \\
\mathrm{P}_{2}\end{array}$ & $\mathrm{P}_{3}$ \\
\cline { 2 - 5 } & \multicolumn{4}{c}{$\left(\mathrm{L} \mathrm{min}^{-1}\right)$} \\
\hline XR 110.02 VK TEEJET & 0,8 & 0,78 & 0,75 & 0,74 \\
AXI ISO 110.03 JACTO & 1,2 & 1,18 & 1,18 & 1,18 \\
AXI ISO 110.04 JACTO & 1,6 & 1,58 & 1,56 & 1,51 \\
AXI ISO 110.05 JACTO & 2,0 & 1,96 & 1,95 & 1,93 \\
\hline
\end{tabular}


$\mathrm{Na}$ avaliação da uniformidade de distribuição de líquido, foi verificado efeito do tipo de ponta em função da altura e pressão de trabalho, corroborando observações de Cunha (2007), que, trabalhando com diferentes pontas de pulverização de jato plano, em diferentes alturas e pressões de trabalho, observou que a uniformidade de distribuição foi afetada pela altura e pressão de trabalho.

Para a ponta XR 110.02 VK TEEJET, constatou-se a melhor condição de trabalho quando a pressão foi de 400 $\mathrm{kPa}$ e a altura da barra de $0,4 \mathrm{~m}$, apresentando um CV de $8,5 \%$. Resultados semelhantes foram obtidos por Cunha e Silva (2010), trabalhando com as pontas TT $110.02 \mathrm{e}$ TTJ60 110.02. Já na pressão de $300 \mathrm{kPa}$ e altura de 0,6 $\mathrm{m}$, o CV foi de $16,68 \%$, considerado inapropriado para a aplicação.

Foi constatada melhor uniformidade de distribuição quando se utilizou a barra a uma altura de $0,4 \mathrm{~m}$, comparada às demais alturas. Os maiores $\mathrm{CVs}$ foram observados a 0,6 $\mathrm{m}$ de altura, indicando que o uso da barra nessa distância pode reduzir a eficiência da pulverização (Quadro 2). Cunha e Silva (2010) afirmam que o aumento da altura melhora o espalhamento no líquido interferindo na uniformidade da sobreposição e, portanto, no coeficiente de variação.

Para a ponta AXI ISO 110.03 JACTO, observou-se que o menor CV foi na pressão de $300 \mathrm{kPa}$ e $0,4 \mathrm{~m}$ de altura do alvo, apresentando um CV de 7,1\%, e o maior
CV foi a $400 \mathrm{kPa}$ de pressão e altura de $0,5 \mathrm{~m}$, com CV de $13,87 \%$. Para as pressões de trabalho de 200 e $300 \mathrm{kPa}$, verificou-se que, com o aumento da altura em relação ao alvo, houve um acréscimo no $\mathrm{CV}$, indicando maior desuniformidade na aplicação. Esta relação não foi constatada na pressão de $400 \mathrm{kPa}$ (Quadro 3).

Resultados diferentes foram obtidos por Figueiredo et al. (2007) avaliando a uniformidade de pontas de pulverização (LD 110.015, LD 110.02, LD 110.03, API 110.015, API 110.02, API 110.03 e JD12P) em função da pressão ( 200, 300 e $400 \mathrm{kPa}$ ) e da altura de trabalho $(0,30 ; 0,40$ e $0,50 \mathrm{~m})$. Com a diminuição da altura de trabalho, em todas as pressões trabalhadas o coeficiente de variação aumentou, exceto na ponta LD 110.015, em que nas pressões de 300 e $400 \mathrm{kPa}$, com a diminuição da altura, houve uma redução no coeficiente de variação. Freitas et al. (2005), trabalhando com a ponta TT 110.02, sob três alturas do alvo $(0,3 ; 0,4$ e $0,5 \mathrm{~m})$ e quatro pressões de trabalho $(100,200,300$ e $400 \mathrm{kPa})$, nas pressões de 100 e $300 \mathrm{kPa}$, com o aumento na altura da barra, observaram que houve melhor uniformidade de distribuição ao longo da barra.

$\mathrm{Na}$ avaliação da uniformidade de distribuição, de acordo com o Quadro 4 utilizando a ponta AXI ISO 110.04 JACTO, a condição que proporcionou melhor uniformidade de aplicação foi na pressão de $300 \mathrm{kPa}$ e $0,4 \mathrm{~m}$ de altura, com CV de 6,3\%. Entretanto, para a altura de $0,6 \mathrm{~m}$, apresentou maiores irregularidades nas

Quadro 2. Coeficiente de variação da ponta XR 110.02 VK TEEJET, em diferentes condições de trabalho

\begin{tabular}{cccc}
\hline & \multicolumn{3}{c}{ Coeficiente de Variação $(\%)$} \\
\cline { 2 - 4 }$(\mathrm{kPa})$ & 0,4 & Altura da barra $(\mathrm{m})$ & \\
& 8,96 & 0,5 & 0,6 \\
\hline 200 & 10,96 & 10,58 & 15,56 \\
300 & 8,52 & 13,24 & 16,68 \\
400 & 12,43 & 16,26 \\
\hline
\end{tabular}

Quadro 3. Coeficiente de variação da ponta AXI ISO 110.03 JACTO, em diferentes condições de trabalho

\begin{tabular}{cccc}
\hline & \multicolumn{3}{c}{ Coeficiente de Variação (\%) } \\
\cline { 2 - 4 }$(\mathrm{kPa})$ & 0,4 & Altura da barra $(\mathrm{m})$ & \\
& 7,77 & 0,5 & 0,6 \\
\hline 200 & 7,10 & 8,48 & 10,38 \\
300 & 9,73 & 9,62 & 13,16 \\
400 & 13,87 & 11,27 \\
\hline
\end{tabular}

\section{REVENG}

541-547 p. Engenharia na Agricultura, ViçOSA - MG, V.19 N.6, NOVEMbro / DEZEMBro 2011 
aplicações, apresentando CV de 13,04\%.

A ponta hidráulica AXI ISO 110.04 JACTO teve o mesmo comportamento da ponta AXI ISO 110.03 JACTO (Quadro 4). Para as pressões de 200 e 300 $\mathrm{kPa}$, verificou-se que o aumento da altura da barra em relação ao alvo aumentou o $\mathrm{CV}$, indicando maior desuniformidade na aplicação. Este comportamento é esperado em situação de campo, pois o aumento na altura da barra, aliado a condições meteorológicas adversas com temperatura elevada, baixa umidade relativa do ar e alta velocidade do vento, acelera a evaporação e a deriva das gotas, corroborando trabalhos de Cunha e Ruas (2006).

Bauer e Raetano (2004), ao avaliarem diferentes modelos de pontas de pulverização (XR, TP e TJ), em diferentes condições de trabalho (altura, pressão e ângulo em relação à horizontal), verificaram que a elevação da barra se reflete na largura de distribuição do líquido e atua negativamente na sobreposição dos jorros. Este pode ser um dos fatores que explicariam a uniformidade de distribuição no presente estudo.

A ponta AXI ISO 110.05 JACTO, na pressão de 200 $\mathrm{kPa}$ e altura de $0,5 \mathrm{~m}$, apresentou menores valores de CV, 7,71\%. Já a $400 \mathrm{kPa}$ e $0,4 \mathrm{~m}$ de altura, mostrou haver maiores irregularidades na deposição de líquidos, com CV de 12,08 \% (Quadro 5).

Foi observado para a ponta AXI ISO 110.05 JACTO que para a mesma altura, com o aumento da pressão de trabalho, houve um aumento do CV, mostrando que para uma maior pressão de trabalho houve uma tendência de aumentar a desuniformidade na aplicação.

Resultados contrários foram obtidos por Ferreira et al. (2007), avaliando a ponta ADGA 110.015, espaçadas $0,5 \mathrm{~m}$ e altura do alvo a $0,5 \mathrm{~m}$, trabalhando nas pressões de 207 e $310 \mathrm{kPa}$. Os CVs obtidos foram 10,83 \% e $3,24 \%$, respectivamente. Viana et al. (2007), avaliando as pontas LA-1JC e SR-1, sob três alturas do alvo $(0,3$; $0,4$ e $0,5 \mathrm{~m})$ e três pressões de trabalho $(100,200$ e 300 $\mathrm{kPa}$ ), observaram que para a ponta SR-1 houve melhor uniformidade de distribuição quando se trabalhou com pressões acima de $200 \mathrm{kPa}$.

A variação na altura da barra proporciona faixas de aplicação diferentes. Além disso, à medida que aumenta a distância entre a ponta de pulverização e a área-alvo, maior será a influência da velocidade do vento sobre as gotas e maior a tendência de deriva. A velocidade do vento eleva o risco de deriva, quanto maior for a altura da barra em relação ao alvo. A pressão de trabalho fornece energia para fracionar o líquido em pequenas gotas. Este é um fator fundamental para regular o tamanho das gotas pulverizadas, visto que, reduzindo a pressão de trabalho, reduz-se a possibilidade de ocorrência de deriva, uma vez que gotas maiores serão formadas. No entanto, se uma ponta é operada abaixo da pressão recomendada, a distribuição do líquido será afetada pela não abertura do ângulo do jato, resultando numa sobreposição inadequada e má distribuição ao longo da barra. A sobreposição entre pontas adjacentes é essencial para assegurar uma boa uniformidade de distribuição do jato de pulverização.

Quadro 4. Coeficiente de variação da ponta AXI ISO 110.04 JACTO, em diferentes condições de trabalho

\begin{tabular}{cccc}
\hline & \multicolumn{3}{c}{ Coeficiente de Variação $(\%)$} \\
\cline { 2 - 4 }$(\mathrm{kPa})$ & 0,4 & Altura da barra $(\mathrm{m})$ & 0,6 \\
\hline 200 & 8,51 & 0,5 & 10,46 \\
300 & 6,30 & 10,07 & 13,04 \\
400 & 7,66 & 9,51 & 9,52 \\
\hline
\end{tabular}

Quadro 5. Coeficiente de variação da ponta AXI ISO 110.05 JACTO, em diferentes condições de trabalho

\begin{tabular}{cccc}
\hline & \multicolumn{3}{c}{ Coeficiente de Variação $(\%)$} \\
\cline { 2 - 4 }$(\mathrm{kPa})$ & 0,4 & Altura da barra $(\mathrm{m})$ & 0,6 \\
\hline 200 & 7,88 & 0,5 & 9,57 \\
300 & 10,95 & 7,71 & 9,74 \\
400 & 12,05 & 8,54 & 12,08 \\
\hline
\end{tabular}




\section{CONCLUSÕES}

- A altura da barra e a pressão de trabalho influenciaram na uniformidade de distribuição de líquido;

- As pontas utilizadas apresentaram coeficiente de variação inferior a $15 \%$, exceto a ponta XR 110.02 VK TEEJET na altura de $0,6 \mathrm{~m}$; e

- Para o coeficiente de variação inferior a $7 \%$, somente a ponta AXI ISO 110.04 JACTO, na pressão de $300 \mathrm{kPa}$ e altura da barra de $0,4 \mathrm{~m}$, proporcionou $\mathrm{CV}$ de $6,30 \%$.

\section{REFERÊNCIAS BIBLIOGRÁFICAS}

ALVARENGA, C.B. Avaliação de pulverizadores hidráulicos de barra na região de Uberlândia - MG. 2009. 70 p. Dissertação (Mestrado em Fitotecnia) - Universidade Federal de Uberlândia, Uberlândia - MG.

BALAN, M.G.; ABI-SAAB, O.J.G.; SILVA, C.G.; RIO, A. Deposição da calda pulverizada por três pontas de pulverização sob diferentes condições meteorológicas. Ciências Agrárias, Londrina, v. 29, n.2, p.293-298, 2008.

BARCELLOS, L.C.; ALMEIDA, R.A.; LEÃO, P.G.; SILVA, J.G. Desenvolvimento e avaliação de um pulverizador de barras a tração humana. Pesquisa Agropecuária Tropical, v.36, n.1, p.6773, 2006.

BAUER, F.C.; RAETANO, C.G. Distribuição volumétrica de calda produzida pelas pontas pulverização XR, TP e TJ sob diferentes condições operacionais. Planta Daninha, Viçosa, v.22, n.2, p.275-284, 2004.

BAUER, F.C.; RAETANO, C.G.; PEREIRA, F.A. R. Padrões de distribuição volumétrica de pontas de pulverização de jato plano 11002 , com e sem indução de ar, sob diferentes espaçamentos e alturas. Engenharia Agrícola, Jaboticabal, v.26, n.2, p.546-551, 2006.

CUNHA, J.P.A.R.; RUAS, R.A.A. Uniformidade de distribuição volumétrica de pontas de jato plano duplo com indução de ar. Pesquisa Agropecuária Tropical, Goiânia, v.36, n.1, p. 61-66, 2006.

CUNHA, J.P.A. Análise da distribuição volumétrica de pontas de pulverização hidráulicas de jato plano. Revista de Ciências Agrárias, Belém, Pará, v.31, n.1, p.233-239, 2007.

CUNHA, P.A.R.; SILVA, R. A. M. Uniformidade de distribuição volumétrica de pontas de pulverização em função da pressão de trabalho e altura da barra. Bioscience Journal, Uberlândia, v. 26, n. 1, p. 5258, 2010.

DeBOER, L.J.; WIENS, E.H. Test procedures for granular applicator performance testing. St. Joseph: ASAE, 1983. (ASAE Paper N_83-1504).

FAO.FOOD AGRICULTURE AND ORGANIZATION. Equipo de aplicación de pesticida para uso en agricultura: equipo impulsado mecánicamente. ROMA, 1997.v.2, 150 p.

FAQIRI, N.I.; KRISHNAN, P. Effect of nozzle pressure and wind condition on spray pattern displacement of RF5 and 110-5R nozzles.St. Joseph: ASAE, 2001. 13p. (ASAE Paper n.011123).

FERNANDES, A.P.; PARREIRA, R.S.; FERREIRA, M.C.; ROMANI, G.N. Caracterização do perfil de deposição e do diâmetro de gotas e otimização do espaçamento entre bicos na barra de pulverização. Engenharia Agrícola, Jaboticabal, v.27, n.3, p.728-733, 2007.

FERREIRA, M.C.; COSTA, G.M.; SILVA, A. R.; TAGLIARI, S.R.A. Fatores qualitativos da ponta de energia hidráulica ADGA 110015 para pulverização agrícola. Engenharia Agrícola, Jaboticabal, v.27, n.2, p.471-8, 2007.

FIGUEIREDO, J.L.A.; TEIXEIRA, M.M.; PICANÇO, M.C.; PINTO, F.A.C.; PRAT, M.H. Avaliação da uniformidade de aplicação e do espectro de gotas de bicos hidráulicos. Revista Ciências Técnicas Agropecuarias, La Habana - 
Cuba, v.16, n.3, p.47-52, 2007.

FREITAS, F.C.L.; TEIXEIRA, M.M.; FERREIRA, L.R.; FERREIRA, F.A.; MACHADO, A.F.L.; VIANA, R.G. Distribuição volumétrica de pontas de pulverização Turbo Teejet 11002 em diferentes condições operacionais. PlantaDaninha, ViçosaMG, v.23, n.1, p.161-167, 2005

ISO. International Organization for Standardization. 1986. Equipment for crop protection - Spraying equipment - Part 2: test methods for agricultural sprayers. ISO, Geneva. 5 p. (ISO 5682/2).

ISO. International Organization for Standardization. 2005. Equipment for crop protection -- Sprayer nozzles -- Colour coding for identification. $4 \mathrm{p}$. (ISO 10625).
RODRIGUES,G. $\quad$ J.; TEIXEIRA, $\quad$ M. T.; FERNANDES FILHO, E. I.; PICANÇO, M. C. Características do fluxo de ar de um pulverizador hidropneumático para aplicação de agroquímicos em plantas arbustivas. Engenharia na Agricultura, Viçosa, MG, v.16, n.2, p.199-207, 2008.

VIANA, R.G.; FERREIRA, L.R.; TEIXEIRA, M.M.; CECON, P.R.; FREITAS, F.C.L.; QUIRINO,A.L.S.; SANTOS, M.V. Características técnicas de pontas de pulverização LA-1JC e SR1. Planta Daninha, Viçosa-MG, v.25, n.1, p.211218, 2007

WOLF, D.D.; SMITH, E. S. Uniformity of seed and fertilizer distribution with a hand-operated spinning spreader.Transactions of the ASAE, St. Joseph, v.22, n.4, 761-2, 1979. 